\title{
Squeezing Effects on the Estimation Precision of Cut off Frequency
}

\author{
Asghar Ullah*, Khalid Khan \\ Department of Physics, Quaid-i-Azam University, Islamabad, Pakistan \\ Email: *asghar.qit@gmail.com
}

How to cite this paper: Ullah, A. and Khan, K. (2021) Squeezing Effects on the Estimation Precision of Cut off Frequency. Journal of Quantum Information Science, 11, 13-23.

https://doi.org/10.4236/jqis.2021.111002

Received: January 1, 2021

Accepted: February 2, 2021

Published: February 5, 2021

Copyright $\odot 2021$ by author(s) and Scientific Research Publishing Inc. This work is licensed under the Creative Commons Attribution International License (CC BY 4.0).

http://creativecommons.org/licenses/by/4.0/

\begin{abstract}
We study the behavior of quantum Fisher information for a qubit probe that is interacting with a squeezed thermal environment. We analyzed the effect of squeezing parameters on the dynamics of quantum Fisher information which affects the optimal precision of the estimation parameter. We show that the squeezed field may offer a significant role in the precise measurement of the parameter cut-off frequency which is linked to the environment correlation time. Our results may be useful in quantum metrology, communication, and quantum estimation processes.
\end{abstract}

\section{Keywords}

Quantum Fisher information (QFI), Squeezed Thermal Environment, Qubit Probe, Cut off Frequency

\section{Introduction}

Quantum Fisher information (QFI) is a core concept in quantum metrology and quantum parameter estimation because it establishes a lower bound for parameters estimation [1] [2] [3] [4] [5]. In recent years, quantum metrology has gained much attention which explains the quantum mechanical effects to achieve precise parameter estimation [1] [6] [7]. Recently, QFI has been widely studied in various fields such as investigation of uncertainty relations [8] [9], estimation of quantum speedup limit [10], characterization of quantum phase transitions [11], detection of entanglement [12] and quantum thermometry [13].

Recently, QFI has been widely used to make high precision measurements of given parameters by using quantum systems and quantum resources. Practically, the quantum systems can never be closed completely and their interaction with the environment cannot be ignored which in turn, transfers the information to 
the quantum system. In such situations, it is essential to study the dynamics of QFI which quantifies the quality of transmitted information. The dynamics of QFI provides information about the different parameters of the environment, i.e. its temperature, time of interaction, and cut-off frequency which is linked to the environment correlation time and indirectly to the coherence time for computation and communication [14].

A common methodology used to enhance the precision of the estimation parameter is to increase the QFI. The higher the QFI is, the higher will be the precision of estimation. Several techniques are used to enhance the QFI for a precise measurement of parameters. The enhancement of QFI is very meaningful because its inverse characterizes the ultimate achievable precision in parameter estimation [15]. Unfortunately, the inevitable coupling of a quantum system with an uncontrolled environment rises the issue of robustness of QFI due to various sources of decoherence [16]. Researches have demonstrated the fragile behavior of QFI which is broken by the environment noise easily [17] [18] [19] [20] [21]. This would be the most liming factor for the applications of QFI in quantum metrology, quantum teleportation, and other quantum tasks. This demands the protection of QFI from decoherence for different estimation procedures.

Different strategies are employed for the enhancement of QFI which includes partial measurements, partially collapsing measurements, and dynamical coupling pulses [22] [23] [24].

In this work, we consider the squeezed thermal environment for the enhancement of QFI. We investigate the dynamics of QFI for a qubit probe interacting with a squeezed thermal environment. This model is well considered to describe the physical systems which include molecular oscillation, the exciton-phonon interaction, and the photosynthesis process [25] [26] [27]. Plenty of studies has been made on this exactly solvable model [28] [29]. Here we adopt that the environment begins with a squeezed thermal state and numerically examines the effects of squeezing strength, relative phase, and temperature on the dynamics of QFI. We notice that squeezing enhances the QFI with respect to time and cut-off frequency which depicts the possibility for precise estimation of cut-off frequency which will increase the environment correlation time.

We arrange the paper as follows: In Section II we present the Hamiltonian of our physical model and briefly review the concept of QFI. The analytical results obtained through numerical simulations and discussion are presented in Section III. Finally, the main results are concluded in Section IV.

\section{Physical Model}

We consider a single qubit probe which is interacting with a squeezed bosonic thermal environment. The Hamiltonian describing the dynamics of composite system can be expressed as

$$
H_{S R}=\frac{1}{2} \omega_{0} \sigma_{z}+\sum_{k} \omega_{k} b_{k}^{\dagger} b_{k}+\sigma_{z} \cdot \sum_{k}\left(g_{k} b_{k}^{\dagger}+g_{k}^{*} b_{k}\right),
$$


where on the right, the parts from left to right, represent the Hamiltonian of the qubit, the reservoir, and the interaction between the two components. In the first part, $\omega_{0}(\hbar=1)$ is the transition energy of the qubit and $\sigma_{z}$ is the Pauli matrix acting on the space of the qubit. In the second part, $\omega_{k}$ is the energy corresponding to the frequency of $k t h$ mode of the reservoir, and $b_{k}\left(b_{k}^{\dagger}\right)$ is the annihilation (creation) operator obeying the usual commutation relations of bosonic operators. In the third part, $g_{k}$ represents the coupling strength of the corresponding mode with the qubit. Since the Hamiltonian of the qubit commutes with the interaction part, the overall effect of $\mathrm{H}$ on the qubit's space is to decohere it. The squeezed thermal state of the environment can be written as [30]

$$
\rho_{R}(0)=S_{\zeta} \rho_{T h} S_{\zeta}^{\dagger},
$$

where $\rho_{T h}=\frac{1}{Z} \mathrm{e}^{-H_{R} / T}$ is the thermal state, $Z=\operatorname{Tr}\left[\mathrm{e}^{-H_{R} / T}\right]$ represents the partition function, $H_{R}$ is the Hamiltonian of environment (second part in Equation (1)) and $\mathrm{T}$ represents the temperature of environment. The the squeezed operator $S_{\zeta}$ for the bosonic environment can be written as

$$
S_{\zeta}=\exp \left[\frac{1}{2} \zeta_{k}^{*} b_{k}^{2}-\frac{1}{2} \zeta_{k}\left(b_{k}^{\dagger}\right)^{2}\right], \zeta_{k}=r_{k} \mathrm{e}^{i \theta_{k}} .
$$

where $\zeta_{k}=r_{k} \mathrm{e}^{\mathrm{i} \theta_{k}}$ with $r \geq 0$ and $\theta \in[0,2 \pi]$ that denote the squeezing and phase parameters respectively. Moving to the interaction picture, the evolved reduced density matrix of the qubit probe is written as:

$$
\rho_{S}(t)=\operatorname{Tr}_{R}\left[U_{I}(t) \rho_{S R}(0) U_{I}^{\dagger}(t)\right],
$$

where $\rho_{S R}=\rho_{S}(0) \otimes \rho_{R}(0)$ is the state of the combined system and is written in the tensor product because the interaction of qubit with the environment is initially considered uncorrelated whereas $\rho_{S}(0)$ is the initial state of the qubit.

The unitary evolution operator in the interaction picture can be written as

$$
U_{I}(t)=\mathrm{e}^{\frac{\sigma_{z}}{2}\left[\alpha_{k} b_{k}^{\dagger}-\alpha^{*} b_{k}\right]}
$$

with $\alpha_{k}=\frac{2 g_{k}}{}\left(1-\mathrm{e}^{i \omega_{k} t}\right)$.

Starting from the pure state of the qubit probe in the form $|\Psi(0)\rangle=\cos \left(\frac{\alpha}{2}\right)|0\rangle+\sin \left(\frac{\alpha}{2}\right)|1\rangle$. Along with this and $\rho_{s}(0)=|\phi(0)\rangle\langle\phi(0)|$, the density matrix for the qubit probe after doing some algebra can be written as

$$
\begin{aligned}
\rho_{s}\left(T, t, \omega_{c}\right)= & \cos ^{2}\left(\frac{\alpha}{2}\right)|0\rangle\left\langle 0\left|+\sin ^{2}\left(\frac{\alpha}{2}\right)\right| 1\right\rangle\langle 1| \\
& +\frac{1}{2} \sin (\alpha) \exp \left[-\Gamma\left(T, t, \omega_{c}\right)\right](|1\rangle\langle 0|+| 0\rangle\langle 1|)
\end{aligned}
$$

where $\Gamma\left(T, t, \omega_{c}\right)$ contains the information of environment which are embedded on the state of qubit and its exponent is written as

$$
\mathrm{e}^{-\Gamma(T, t)}=\sum_{k}\left\langle\mathrm{e}^{\left(\beta_{k} b^{\dagger}-\beta^{*} b_{k}\right)}\right\rangle
$$

Note that the above expression for $\mathrm{e}^{-\Gamma\left(T, t, \omega_{c}\right)}$ is the characteristic function of 
Wigner representation of $\rho_{R}(0)$, which is a squeezed thermal state of all modes [31] [32].

Thus we get

$$
\Gamma(T, t)=\sum_{k} \frac{1}{2}\left|\beta_{k}(t)\right|^{2} \operatorname{coth}\left(\frac{\omega_{k}}{2 T}\right)
$$

where

$$
\beta_{k}(t)=\alpha_{k} \cosh r_{k}+\alpha_{k}^{*} \mathrm{e}^{i \theta_{k}} \sinh r_{k}
$$

Hence the decay factor can be written as

$\Gamma(T, t)=\sum_{k} 4\left|g_{k}\right|^{2}\left(\frac{1-\cos \omega_{k} t}{\omega_{k}^{2}}\right)\left[\cosh 2 r_{k}-\cos \left(\theta-\omega_{k} t\right) \sinh 2 r_{k}\right] \operatorname{coth}\left(\frac{\omega_{k}}{2 T}\right)$.

For continuous reservoir modes, the summation over $\left|g_{k}\right|^{2}$ changes into integral and introducing the ohmic spectral density $J(\omega)$ that is

$\sum_{k}\left|g_{k}\right|^{2} \rightarrow \int J(\omega) \mathrm{d} \omega$. Applying continuous mode approximations, the decay function can explicitly be written as

$$
\Gamma(T, t)=\int J(\omega)\left(\frac{1-\cos \omega t}{\omega^{2}}\right)[\cosh 2 r-\cos (\theta-\omega t) \sinh 2 r] \operatorname{coth}\left(\frac{w}{2 T}\right) .
$$

The above decoherence function depends on temperature and also on the spectral density $J\left(\omega, \omega_{c}\right)$ of the coupling frequencies of reservoir, so for ohmic family, it is given by

$$
J\left(\omega, \omega_{c}\right)=\frac{\omega^{s}}{\omega_{c}^{s-1}} \exp \left\{-\frac{\omega}{\omega_{c}}\right\}
$$

where $\omega_{c}$ is the cut off frequency which describes a natural boundary in frequency response of the system and $s$ is the true positive number that controls the behavior of spectral density at low frequencies and is called ohmicity parameter. Various values of the ohmicity parameter usually correspond to radically different types of dynamics, and therefore, it would be greatly recommendable to have an estimation scheme for their precise characterization. This dimensionless parameter classifies the environment into three classes, i.e. ohmic $(s=1)$, sub-ohmic $(s=0.5)$ and super-ohmic $(s=3)$.

\section{Quantum Fisher Information}

QFI has different useful versions but in this paper we will consider symmetric logarithmic derivative based QFI [1] [7] [33] [34]. The QFI for any parameter $\theta$ is defined as

$$
I_{\theta}=\operatorname{Tr}\left[\rho L_{\theta}^{2}\right]
$$

where $L_{\theta}$ is found by $\frac{\partial \rho}{\partial \theta}=\frac{1}{2}\left[\rho L_{\theta}+L_{\theta} \rho\right]$.

The QFI with respect to a parameter $\beta$, for a spectrally decomposed density matrix $\rho(\beta)=\sum_{i} \lambda_{i}(\beta)\left|\phi_{j}(\beta)\right\rangle\left\langle\phi_{i}(\beta)\right|$ matrix can be expressed as [35]

$$
I\left(\omega_{c}\right)=\sum_{s} \frac{\left(\partial_{\omega_{c}} \lambda_{s}\right)^{2}}{\lambda_{s}}+2 \sum_{l \neq m} \frac{\left(\lambda_{l}-\lambda_{m}\right)^{2}}{\lambda_{l}+\lambda_{m}}\left|\left\langle\phi_{l} \mid \partial_{\omega_{c}} \phi_{m}\right\rangle\right|^{2},
$$


where the first part depends only on the eigenvalues and corresponds to classical Fisher information. The second part, in addition to eigenvalues, also depends on eigenvectors and is thus quantum mechanical in nature. A measurement on the final density matrix is said to be optimal for which the QFI reduces to classical Fisher Information. Moreover, being used as an estimation tool for different purposes [3] [36], it is required to search for the parameters of the system's space that maximizes QFI. The eigenvalues and eigenfunctions of the density matrix in Equation (6) are calculated as,

$$
\lambda^{ \pm}=\frac{1}{2}\left(1 \pm \exp \left[\Gamma\left(T, t, \omega_{c}\right)\right]\right) \kappa(\Gamma, \alpha)
$$

and

$$
\Phi^{ \pm}\left(T, t, \omega_{c}\right)=\left[\exp \left\{\Gamma\left(T, t, \omega_{c}\right)\right\} \cot \theta \pm \csc \theta \kappa(\Gamma, \alpha)\right]|0\rangle+|1\rangle,
$$

with function $\kappa(\Gamma, \alpha)=\left[\exp \left\{2 \Gamma\left(T, t, \omega_{c}\right)\right\} \cos ^{2} \alpha+\sin ^{2} \alpha\right]^{1 / 2}$ After substituting Equations (15) and (16) in (14), expression for the QFI reads to

$$
I\left(T, t, \omega_{c}\right)=\frac{\sin ^{2}(\alpha)\left[\partial_{\omega_{c}} \Gamma\left(T, t, \omega_{c}\right)\right]^{2}}{\mathrm{e}^{2 \Gamma\left(T, t, \omega_{c}\right)}-1}
$$

The QFI is maximum when $\alpha=\pi / 2$ and the corresponding initial pure state of qubit probe is $|+\rangle=\frac{1}{\sqrt{2}}(|0\rangle+|1\rangle)$. By considering this initial state of the qubit, the function $\kappa(\Gamma, \alpha)=1$ and eigenvectors become temperature independent and the second part of Equation (14) vanishes.

\section{Numerical Results and Discussion}

In this section, we will present the results by using the numerical simulations of Equation (17) due to a complex form of $\Gamma\left(T, t, \omega_{c}\right)$. Using these results, we will investigate the effects of different parameters of the environment on the dynamics of QFI. For simplicity, the interaction time thas been scaled out, i.e., one unit of time is given by the inverse of the coupling constant $\mathrm{g}$.

Figure 1 describes the behavior of QFI with respect to the cut-off frequency $\omega_{c}$ (upper panel) and the interaction time $t$ (lower panel) for three different choices of the temperature, squeezing parameter $r$ (first column) and the phase parameter $\theta$ (second column) for the sub-ohmic regime with $(s=0.5)$. The QFI increases as the value of the squeezing parameter $r$ is increased as a function of cut-off frequency $\omega_{c}$ and it can be observed that the peak value is highest at $r=2$. From the plot of Figure $1(\mathrm{~b})$ and Figure 1(c), it is obvious that, at $r=0.5$, the QFI is not considerably affected by squeezing the field and the rate of increase and decrease at either side of the peaks is moderate and the peaks are broadened that shows, more time is needed for information to be imprinted on the qubit state. However, upon increasing the squeezing strength such that at $r=1.5$, the broadness of both peaks diminishes but it is more notable when QFI is plotted against time and the peak sharpened at $r=2$. The peaks are shifted 


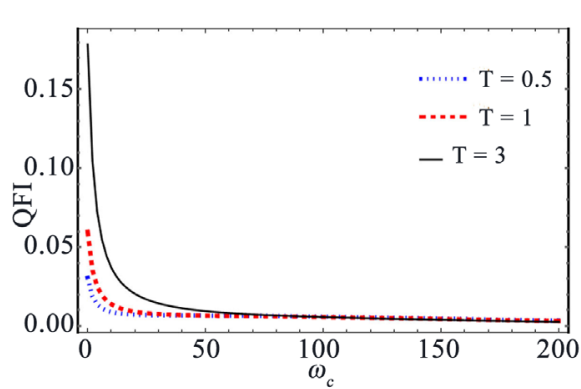

(a)

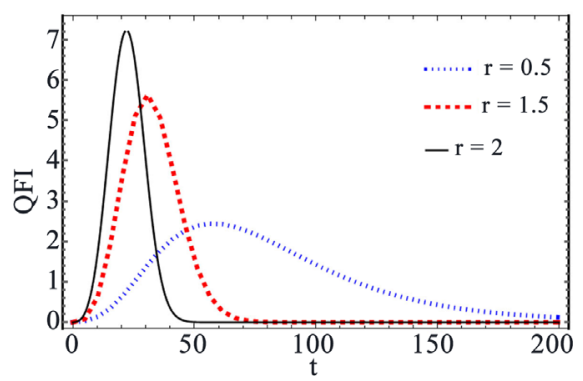

(c)

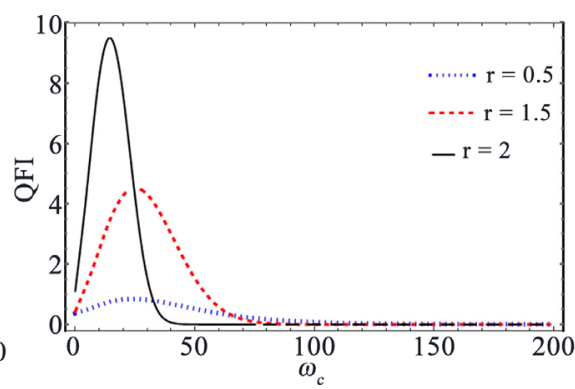

(b)

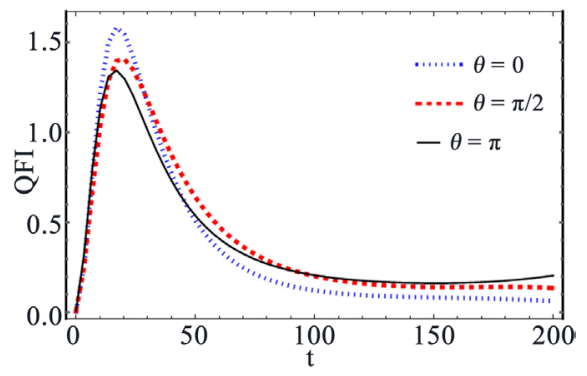

(d)

Figure 1. (Colour online) QFI versus cut-off frequency $\omega_{c}$ (upper panel) and QFI verses time $t$ (lower panel) at different values of temperature, squeezing parameter and phase parameter for sub ohmic $(s=0.5)$ case. Here the other parameters used are: (a) $t=0.1, \quad \theta=0.1, \quad r=0.1$; (b) $t=0.1, T=0.01, \theta=0.1$; (c) $T=0.01, \theta=0.1$, $\omega_{c}=0.5$ and $(\mathrm{d}) \quad T=0.01, r=0.1, \omega_{c}=0.5$.

towards the origin which shows that maximum information about the parameter cut-off frequency is encoded onto the state of qubit at these peaks. This type of behavior of QFI is very advantageous in various estimation procedures.

For different values of phase parameter, $\theta$ (Figure 1(d)), the QFI increases and reaches its maximum value at $\theta=0$ and then oscillates between the upper $(\theta=0)$ and lower $(\theta=\pi)$ bound. The QFI reaches its maximum value sooner but decreases gradually and ultimately saturates for each chosen value of $\theta$. It was observed from numerical simulations that the value of QFI is the same when $\theta$ is odd and even integral multiple of $\pi$, also an odd integral multiple of $\frac{\pi}{2}$. When QFI is plotted against cut off frequency for different values of the temperature of the environment (Figure 1(a)), the QFI decreases sharply from a maximum value and approaches to infinity at $\omega_{c}=0$. At higher temperature for $T=3$, QFI decays rapidly from maximum to minimum value while at lower temperature $T=0.5$, QFI decays slowly which shows that the information loss from the environment is slow as compared to high temperature. Hence it is concluded that the two peaks where the QFI is maximum i.e. Figure 1(b) and Figure $1(\mathrm{c})$, the maximum information about the cut off frequency is encoded on the state of the qubit and it will increase the optimal value for the projective measurements that are taken over the state of the qubit. These results show the strong dependence of QFI on squeezing strength $r$ rather than temperature and phase parameter. The theory of quantum metrology suggests that an increase in 
QFI means that the precision in the estimation of parameters is improved. Since squeezed state has reduced uncertainty, therefore squeezed thermal environments may play efficient role in quantum metrology.

Figure 2 depicts the behavior of QFI for the ohmic regime $(s=1)$ with the same set of values of the parameters which were selected in Figure 1. It can be clearly seen that the QFI with respect to cut-off frequency and time increases with the increase in squeezing parameter $r$ and the peak again reaches its maximum value at $r=2$. However, it can be observed that in the ohmic case the peak of QFI with respect to cut off frequency is more broadened as compared to the sub-ohmic case whereas the peak of QFI with respect to time (Figure 1(c)) is shifted towards the left and less broadened. This shows that in the ohmic case, the QFI is maximum compared to the sub-ohmic case but the time required to get information about cut frequency from the environment is reduced this time. The peak values of QFI against time for different values of $\theta$ are highly reduced as compared to the case of the sub-ohmic regime. The QFI increases first, then decreases gradually, and eventually saturates for each value of $\theta$.

Finally we study the dynamics of QFI for a super-ohmic regime $(s=3)$ by considering the effects of squeezing parameters on QFI. In Figure 3, we have plotted QFI against cut off frequency and time for the same set of values of parameters as selected earlier in sub-ohmic and ohmic case. It can be noted that QFI against $\omega_{c}$ increases upon increasing the value of $r$ and the QFI is once again maximum for $r=2$, however, one of the interesting facts is that the peak value of QFI is highly reduced as compared to sub ohmic and ohmic regimes. In the super-ohmic regime, the behavior of QFI is relatively different from previously discussed cases. For $r=0.5$, the peak value of QFI is very less broadened as compared to previous results and for $r=1.5$, the QFI reaches its maximum value rapidly, and then, it also reaches zero very immediately. The same is the case when squeezing strength is further enhanced $(r=2)$. In both cases, the sharpness of the peak is the same which shows that very less time is required to obtain information from the environment. Looking at Figure 1(c) and Figure 1(d), one can clearly observe that the Peak of QFI is more shifted towards the left and QFI reaches its maximum value sooner as compared to Figure 1 and Figure 2. Moreover, this shows that in a super ohmic case, the higher values of QFI are achievable when the interaction of qubit with its environment is longer and it will provide easiness to estimate the cut-off frequency more precisely. Therefore, it is concluded that the optimal estimation precision for the desired parameter cannot be obtained easily in the super ohmic case. One of the important different results can be drawn from Figure 3(a), where at some specific cut off frequency, the curve with low temperature gives more information because there is no saturation at low $\mathrm{T}$ while information saturates at high temperature. Further investigation shows that the peaks of QFI against $\theta$, i.e. Figure $3(d)$, are less broadened and no saturation effect is observed for given values of the phase parameter $\theta$. 


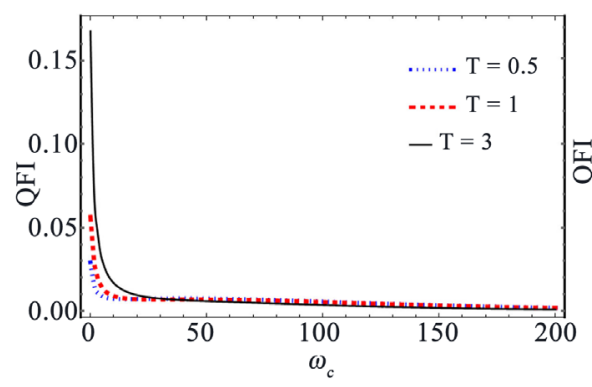

(a)

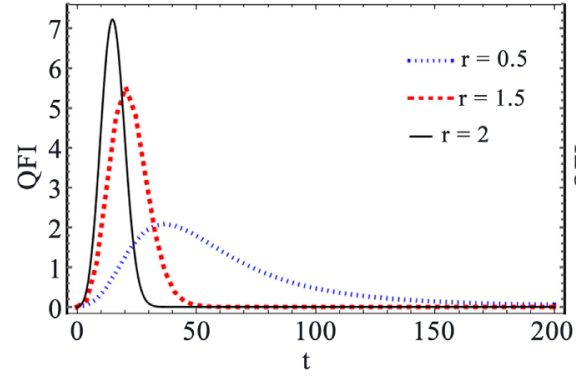

(c)

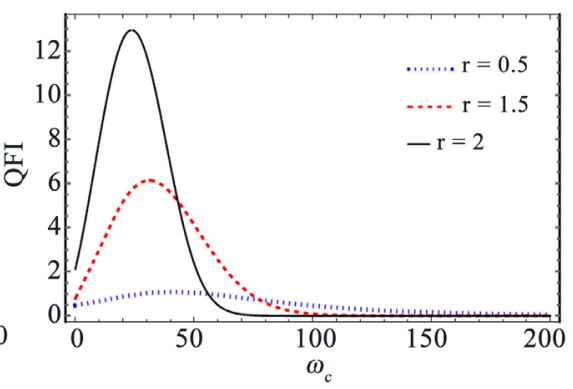

(b)

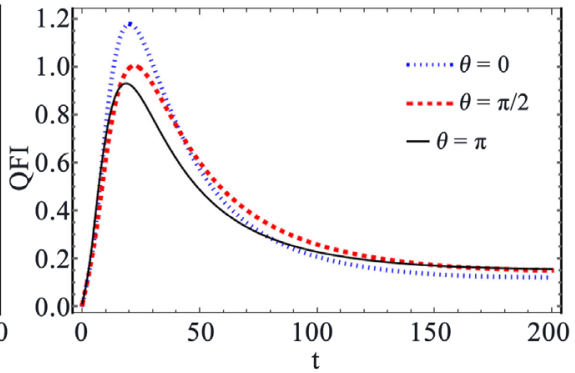

(d)

Figure 2. (Colour online) QFI versus cut-off frequency $\omega_{c}$ (upper panel) and QFI verses time $t$ (lower panel) at different values of temperature, squeezing parameter and phase parameter for ohmic $(s=1)$ case. Here the other parameters are: (a) $t=0.1$, $\theta=0.1, r=0.1$; (b) $t=0.1, T=0.01, \theta=0.1$; (c) $T=0.01, \theta=0.1, \omega_{c}=0.5$ and (d) $T=0.01, r=0.1, \omega_{c}=0.5$.

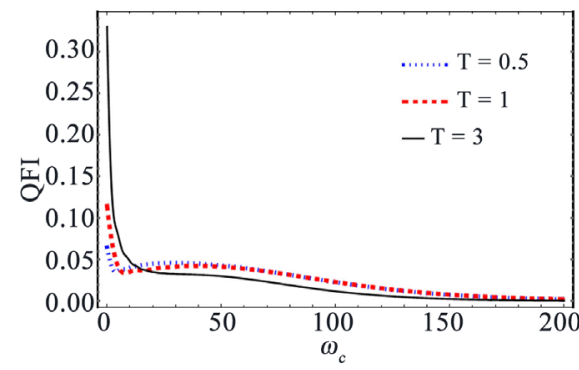

(a)

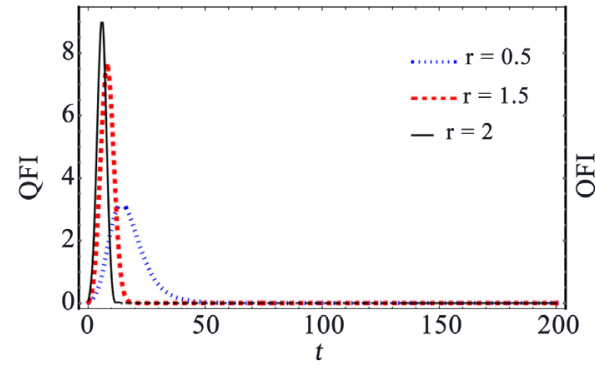

(c)

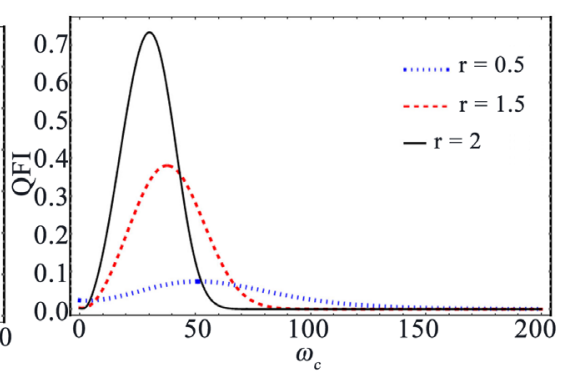

(b)

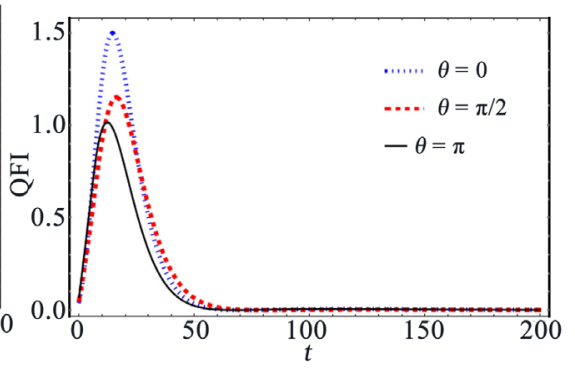

(d)

Figure 3. (Colour online) QFI versus cut-off frequency $\omega_{c}$ (upper panel) and QFI verses time $t$ (lower panel) at different values of temperature, squeezing parameter and phase parameter for super ohmic $(s=3)$ case. Here the other parameters are: (a) $t=0.1$, $\theta=0.1, r=0.1$; (b) $t=0.1, T=0.01, \theta=0.1$; (c) $T=0.01, \theta=0.1, \omega_{c}=0.5$ and (d) $T=0.01, r=0.1, \omega_{c}=0.5$. 


\section{Conclusion}

We have theoretically studied the dynamics of quantum Fisher information for a qubit probe that is interacting with a squeezed thermal environment. We have examined the behavior of QFI through numerical simulations and found that how QFI is maximized through the initial state of the qubit probe. Furthermore, we have described the effects of squeezing parameters on the QFI to extract maximum information about $\omega_{c}$ that is imprinted onto the state of the qubit. We extended our results for three types of environments, i.e. sub-Ohmic, Ohmic, and super-Ohmic environments. It was found that for all three cases squeezing enhances the QFI which means that the precision of the estimation parameter is improved. When QFI is investigated with respect to time and cut off frequency, it is found that we have more time required to obtain optimal precision of the parameter, i.e. cut off frequency in sub-Ohmic and Ohmic case. However, in the super-Ohmic case, the QFI decays rapidly which shortens the interaction time for optimal measurements due to decoherence effects. Our results show that more squeezed fields lead to interesting and nontrivial effects on the optimal precision of cut off frequency. These results can play a significant role in quantum metrology, communication, and quantum estimation processes.

\section{Conflicts of Interest}

The authors declare no conflicts of interest regarding the publication of this paper.

\section{References}

[1] Helstron, C.W. (1976) Quantum Detection and Estimation Theory. Academic, New York.

[2] Holevo, A.S. (2001) Statistical Structure of Quantum Theory. Springer, Berlin. https://doi.org/10.1007/3-540-44998-1

[3] Javed, M., Khan, S. and Ullah, S.A. (2018) Characterization of Classical Static Noise via Qubit as Probe. Quantum Information Processing, 17, 53. https://doi.org/10.1007/s11128-018-1817-x

[4] Paris, M.G.A. (2014) Quantum Probes for Fractional Gaussian Processes. Physica A, 413, 256-265. https://doi.org/10.1016/j.physa.2014.06.052

[5] Benedetti, C. and Paris, M.G.A. (2014) Characterization of Classical Gaussian Processes Using Quantum Probes. Physics Letters A, 378, 2495-2500. https://doi.org/10.1016/j.physleta.2014.06.043

[6] Liu, J. and Yuan, H.D. (2017) Control-Enhanced Multiparameter Quantum Estimation. Physical Review A, 96, Article ID: 042114. https://doi.org/10.1103/PhysRevA.96.042114

[7] Braustein, S.L. and Caves, C.M. (1994) Statistical Distance and the Geometry of Quantum States. Physical Review Letters, 72, 3439. https://doi.org/10.1103/PhysRevLett.72.3439

[8] Luo, S. (2000) Quantum Fisher Information and Uncertainty Relations. Letters in Mathematical Physics, 53, 243-251. https://doi.org/10.1023/A:1011080128419

[9] Giblisco, P., Imparato, D. and Isola, T. (2007) Uncertainty Principle and Quantum 
Fisher Information. Journal of Mathematical Physics, 48, Article ID: 072109. https://doi.org/10.1063/1.2748210

[10] Taddei, M.M., Escher, B.M., Davidovich, L. and de Mtos Filho, R.L. (2013) Quantum Speed Limit for Physical Processes. Physical Review Letters, 110, Article ID: 050402. https://doi.org/10.1103/PhysRevLett.110.050402

[11] Invernizzi, C., Korbman, M., Venuti, L.C. and Paris, M.G.A. (2008) Quantum Criticality as a Resource for Quantum Estimation. Physical Review A, 78, Article ID: 042105. https://doi.org/10.1103/PhysRevA.78.042105

[12] Boixo, S. and Monaras, A. (2008) Precision Measurement of Neutrino Oscillation Parameters with Kam-LAND. Physical Review Letters, 100, Article ID: 221803. https://doi.org/10.1103/PhysRevLett.100.221803

[13] Razavian, S., Benedetti, C., Bina, M., Akbari, Y. and Paris, M.G.A. (2019) Quantum Thermometry by Single-Qubit Dephasing. European Physical Journal Plus, 134, 284. https://doi.org/10.1140/epjp/i2019-12708-9

[14] Benedetti, C., Sehdaran, F.S., Zandi, M.H. and Paris, M.G.A. (2018) Two-Qubit Quantum Probes for the Temperature of an Ohmic Environment. Physical Review $A$, 97, Article ID: 012126. https://doi.org/10.1103/PhysRevA.97.012126

[15] Braunstein, S.L. and Carlton, M.C. (1994) Statistical Distance and the Geometry of Quantum States. Physical Review Letters, 72, 3439.

https://doi.org/10.1103/PhysRevLett.72.3439

[16] Breuer, H.P. and Petroccione, F. (2002) The Theory of Open Quantum Systems. Oxford University Press, Oxford.

[17] Kolodyynski, J. and Demkowicz-Dobrzanski, R. (2010) Phase Estimation without a Priori Phase Knowledge in the Presence of Loss. Physical Review A, 82, Article ID: 053804. https://doi.org/10.1103/PhysRevA.82.053804

[18] Ma, J., Huang, Y.X., Wang, X. and Sun, C.P. (2011) Quantum Fisher Information of the Greenberger-Horne-Zeilinger State in Decoherence Channels. Physical Review $A$, 84, Article ID: 022302. https://doi.org/10.1103/PhysRevA.84.022302

[19] Berrada, K., Abdel-Khalek, S. and Obada, A.S. (2012) Quantum Fisher Information for a Qubit System Placed inside a Dissipative Cavity. Physics Letters A, 376, 1412-1416. https://doi.org/10.1016/j.physleta.2012.03.023

[20] Zhang, Y.M., Li, X.Y., Yang, W. and Jin, G.R. (2013) Quantum Fisher Information of Entangled Coherent States in the Presence of Photon Loss. Physical Review A, 88, Article ID: 043832. https://doi.org/10.1103/PhysRevA.88.043832

[21] Li, Y.L., Xiao, X. and Yao, Y. (2015) Classical-Driving-Enhanced Parameter-Estimation Precision of a Non-Markovian Dissipative Two-State System. Physical Review A, 91, Article ID: 052105. https://doi.org/10.1103/PhysRevA.91.052105

[22] Xiao, X., Yao, Y., Zhong, W.J., Li, Y.L. and Xie, Y.M. (2016) Enhancing Teleportation of Quantum Fisher Information by Partial Measurements. Physical Review A, 93, Article ID: 012307. https://doi.org/10.1103/PhysRevA.93.012307

[23] Pan, F., Qiu, L. and Liu, Z. (2017) The Complementarity Relations of Quantum Coherence in Quantum Information Processing. Scientific Reports, 7, Article No. 43919. https://doi.org/10.1038/srep43919

[24] Tan, Q.-S., Huang, Y., Yin, X., Kuang, L. and Wang, X. (2013) Enhancement of Parameter Estimation Precision in Noisy Systems by Dynamical Decoupling Pulses. Physical Review A, 87, Article ID: 032102. https://doi.org/10.1103/PhysRevA.87.032102

[25] You, Y.-N. and Li, S.-N. (2018) Entropy Dynamics of a Dephasing Model in a 
Squeezed Thermal Bath. Physical Review A, 97, Article ID: 012114. https://doi.org/10.1103/PhysRevA.97.012114

[26] May, V. and Kluhn, O. (2000) Charge and Energy Transfer Dynamics in Molecular Systems: A Theoretical Introduction. Wiley-VCH, Berlin.

[27] Dong, H., Wang, D.-W. and Kim, M.B. (2017) How Isolated Is Enough for an "Isolated" System in Statistical Mechanics?

[28] Agarwal, G.S. (2012) Quantum Optics. Cambridge University Press, Cambridge.

[29] Schaller, G. (2014) Open Quantum Systems Far from Equilibrium. Springer, New York. https://doi.org/10.1007/978-3-319-03877-3

[30] Banerjee, S. and Ghosh, R. (2007) Dynamics of Decoherence without Dissipation in a Squeezed Thermal Bath. Journal of Physics A: Mathematical and Theoretical, 40, 13735. https://doi.org/10.1088/1751-8113/40/45/014

[31] Zhong, W., Sun, Z., Ma, J., Wang, X.G. and Nori, F. (2013) Fisher Information under Decoherence in Bloch Representation. Physical Review A, 87, Article ID: 022337. https://doi.org/10.1103/PhysRevA.87.022337

[32] Benedetti, C., Sehdaran, F.S., Zandi, M.H. and Paris, M.G.A. (2018) Quantum Probes for the Cutoff Frequency of Ohmic Environments. Physical Review A, 97, Article ID: 012126. https://doi.org/10.1103/PhysRevA.97.012126

[33] Song, H., Luo, S. and Hong, Y. (2015) Quantum Criticality from Fisher Information. Physical Review A, 91, Article ID: 042110. https://doi.org/10.1103/PhysRevA.91.042110

[34] Zheng, Q., Ge, L., Yao, Y. and Zhi, Q. (2015) Enhancing Parameter Precision of Optimal Quantum Estimation by Direct Quantum Feedback. Physical Review A, 91, Article ID: 033805. https://doi.org/10.1103/PhysRevA.91.033805

[35] Bennett, C.H., Bernstein, H.J., Popescu, S. and Schumacher, B. (1996) Concentrating Partial Entanglement by Local Operations. Physical Review A, 53, 2046-2052. https://doi.org/10.1103/PhysRevA.53.2046

[36] Pennini, F. and Plastino, A. (2004) Escort Husimi Distributions Fisher Information and Nonextensivity. Physics Letters A, 326, 20-26.

https://doi.org/10.1016/j.physleta.2004.04.024 